\title{
EXPECTATIVA E EXPERIENNCIA DO PROCESSO PARTURITIVO EM MULHERES ATENDIDAS EM UNIDADE BÁSICA DE SAÚDE
}

\section{Eliete dos Reis Carvalho}

Enfermeira, Mestre em Promoção da Saúde pelo Programa de Pós-Graduação em Promoção da Saúde do Centro Universitário de Maringá - UNICESUMAR, Brasil.

\section{Roney Alan Nogueira}

Enfermeiro, Mestrando em Promoção da Saúde pelo Programa de Pós-Graduação em Promoç̧ão da Saúde do Centro Universitário de Maringá - UNICESUMAR, Brasil.

\section{Andréa Grano Marques}

Psicóloga, Docente Titular do Departamento de Psicologia e do Programa de Pós-Graduação em Promoção da Saúde do Centro Universitário de Maringá - UNICESUMAR. Bolsista do Programa de Produtividade em Pesquisa do Instituto Cesumar de Ciência, Tecnologia e Inovação - ICETI, Brasil.

\section{Sônia Silva Marcon}

Enfermeira, Docente Titular do Departamento de Enfermagem e do Programa de Pós-Graduação em Enfermagem da Universidade Estadual de Maringá UEM, Brasil.

\section{Rute Grossi Milani}

Psicóloga, Docente Titular do Departamento de Psicologia e dos Programas de Pós-Graduação em Promocạ̃o da Saúde e em Tecnologias Limpas do Centro Universitário de Maringá - UNICESUMAR Bolsista do Programa de Produtividade em Pesquisa do Instituto Cesumar de Ciência, Tecnologia e Inovação - ICETI, Brasil.
RESUMO: O objetivo deste estudo foi analisar a expectativa da gestante em relação ao parto no final da gestação e a vivência parturitiva. A amostra foi composta por 16 mulheres que se encontravam no terceiro trimestre de gestação, primíparas e que realizaram o prénatal em Unidade Básica de Saúde. Foram realizadas entrevistas semiestruturadas no terceiro trimestre de gestação e após o parto. Utilizou-se a análise de conteúdo para o tratamento e discussão dos dados. O estudo identificou que o parto vaginal foi a via preferida pelas gestantes, entretanto a cesárea eletiva foi a principal via de parto realizada pelos obstetras, mesmo em mulheres cuja preferência era a via de parto vaginal. Concluiu-se que é preciso investir no esclarecimento da população médica, das gestantes e dos seus familiares de que a cesárea eletiva expõe desnecessariamente os recém-nascidos a riscos e complicações neonatais, por meio de programas e de ações de promoção à saúde do grupo materno-infantil.

PALAVRAS-CHAVE: Assistência pré-natal; Gravidez; Parto normal; Promoção da saúde.

\section{EXPECTATION AND EXPERIENCE IN THE BIRTH PROCESS IN FEMAIES ATTENDED BY THE BRAZILIAN NHS}

ABSTRACT: Expectations of pregnant females with regard to birth at the end of pregnancy and parturition living are evaluation. Sample was composed of 16 primipara females at the third pregnancy trimester, with prenatal exams undertake in NHS units. Half-structured interviews were undertaken in the third semester of pregnancy and after birth. Content analysis was employed for data treatment and discussion. The study identified that vaginal birth was preferred by pregnant females, although elective caesarian section was the main method by obstetricians, even in females with the first preference. Investment in education of the medical professionals, pregnant females and their relatives on elective caesarean section is necessary to denounce the risks and complication for newly born children, through programs and health promotion activities for the mother-child health.

KEY WORDS: Prenatal care; Pregnancy; Natural childbirth; Health promotion.

\section{INTRODUÇÃO}

O desejo de ser mãe é idealizado durante muitos anos no ciclo de vida da maioria das mulheres, sendo que a descoberta da gravidez representa o início tanto da concretização deste ideal quanto da reestruturação dos papéis femininos frente à maternidade. As
Autor correspondente

Andréa Grano Marques

andreagrano298@hotmail.com 
modificações corporais, psicológicas e sociais que ocorrem na gestação podem desencadear medos e ansiedades que se intensificam ao longo do período gestacional com a aproximação do parto ${ }^{1}$.

O desfecho gestacional contribui para a saúde materna e infantil, pois as intercorrências negativas colocam em risco a saúde da mulher e do bebê e interferem na formação do vínculo entre ambos ${ }^{1}$. Os sentimentos negativos em relação ao parto e a insatisfação com a via de parto realizada podem interferir na disponibilidade materna para atender às necessidades do recém-nascido². É importante considerar também que o parto simboliza a transição do status de mulher para o de mãe, o que explica a sua importância como ritual de transformação e de passagem ${ }^{3}$.

O parto foi influenciado ao longo do tempo pelas mais distintas práticas e costumes que envolviam o ato de nascer, portanto o desfecho gestacional se caracteriza como um evento determinado historicamente ${ }^{4}$. Até o final do século XVIII, o parto era um acontecimento natural realizado no próprio domicílio com o acompanhamento e auxílio das parteiras e na falta delas algum membro da própria família, geralmente uma mulher, mãe ou sogra, realizava o parto ${ }^{4}$.

A obstetrícia surgiu, como especialidade médica, na Europa na primeira metade do século XIX modificando o perfil dos partos naturais e dando origem às práticas obstétricas atuais ${ }^{5}$. O parto e o nascimento, que antes eram vistos como um evento fisiológico e feminino, começaram a ser concebidos como um procedimento médico e verdadeiras campanhas foram realizadas por obstetras com o propósito de transformar o parto em um evento que pudesse ser controlado por eles e circunscrito às maternidades, incluindo a noção de risco e de complicações como regra e não mais exceção, neste modelo tecnocrático, a mulher deixou de ser a protagonista, cabendo ao médico a tomada das decisões relacionadas ao parto 5 .

Neste contexto, o parto cesáreo, que antes era considerado um procedimento cirúrgico de exceção e indicado em situações de risco de vida real para a gestante ou para o feto, se tornou um procedimento programado com dia e hora para acontecer sem a identificação médica de risco definido ${ }^{6}$. É indiscutível a importância do parto cesariano para os casos em que existe risco para a mãe ou para o bebê, pois as cesáreas podem efetivamente reduzir a mortalidade de mães e bebês quando realizadas com indicação correta 7 .

$\mathrm{O}$ índice de parto cirúrgico no $\mathrm{Brasil}^{8}$ vem aumentando significativamente, atingindo $57 \%$ dos partos realizados no território nacional pelo Sistema Único de Saúde. Este índice se encontra longe de alcançar o valor de parto cirúrgico recomendado pela Organização Mundial da Saúde que corresponde a faixa entre 10 a $15 \%$ do número total de partos realizados ${ }^{9}$. No setor privado o percentual de partos cirúrgicos realizado é mais expressivo, pois atinge $80 \%$ dos partos realizados na saúde suplementar sem justificativa clínica para a escolha do parto cesáreo9.

A Organização Mundial da Saúde ${ }^{10}$ compilou informações sobre a assistência ao parto normal e definiu as condutas obstétricas recomendadas e àquelas que necessitam ser realizadas com cautela pela falta de comprovação de seus benefícios, pelas danosas ou prejudiciais que devem ser abolidas e pelas práticas inadequadas. Baseado nesse documento, no ano 2000, o Ministério da Saúde lançou o Programa de Humanização do Pré-natal e Nascimento a fim de garantir as recomendações e direitos da mulher no ciclo gravídicopuerperal ${ }^{11}$.

Com propósitos voltados especificamente ao processo gravídico-puerperal o Programa de Humanização do Pré-natal e Nascimento preconizou a assistência integral e qualificada na gestação, parto e puerpério, para diminuir o percentual de partos cirúrgicos realizados e restringir a utilização de práticas inadequadas que pudessem comprometer a saúde da mulher e do bebê. ${ }^{12}$ Este programa evidenciou a necessidade de práticas humanizadas na atenção obstétrica e neonatal como elemento primordial para o adequado acompanhamento do parto e puerpério ${ }^{12}$.

A gestação e o parto são resultantes de uma complexa rede de determinantes biológicos, psicológicos e sociais, portanto a assistência pré-natal deve abordar tanto os aspectos clínicos-obstétricos quanto os aspectos subjetivos da mulher. Considerando que o desfecho gestacional é a principal preocupação da gestante ao final do período gestacional, o objetivo desta pesquisa foi 
analisar a expectativa da gestante em relação ao parto no último trimestre da gestação e a vivência parturitiva.

\section{METODOLOGIA}

Trata-se de uma pesquisa exploratória-descritiva, longitudinal, com abordagem qualitativa. A amostra foi composta por 16 mulheres que se encontravam no terceiro trimestre de gestação, primíparas, com 18 anos ou mais e que realizavam a assistência pré-natal em uma Unidade Básica de Saúde. Foram excluídas do estudo gestantes multíparas, em função de possível interferência da experiência do parto anterior.

As gestantes foram selecionadas pela técnica de amostragem intencional nos dias das consultas do pré-natal, e enquanto aguardavam o atendimento eram informadas sobre o objetivo e etapas da pesquisa, pois foram realizadas duas entrevistas, a primeira no último trimestre de gestação, na própria Unidade Básica de Saúde, e a segunda após o parto, na residência. Após o consentimento e a assinatura do Termo de Consentimento Livre e Esclarecido (TCLE) a gestante foi incluída no estudo e a primeira entrevista semiestruturada foi realizada.

Após 20 dias da data prevista do parto foi realizado contato telefônico com as participantes para a confirmação da realização do parto e agendamento para a realização da segunda entrevista semiestruturada. As perguntas versaram sobre as expectativas em relação ao parto e à história obstétrica e as entrevistas semiestruturadas foram gravadas em áudio, com a anuência das participantes, e transcritas na íntegra pela pesquisadora. A coleta de dados foi realizada no período de abril a setembro de 2017.

As transcrições foram submetidas à análise de conteúdo segundo o método de $\operatorname{Bardin}^{13}$. Desta forma, os dados foram agrupados por respostas afins e decodificados em categorias para o tratamento dos resultados e posterior análise. Esta pesquisa seguiu os preceitos éticos conforme resolução no 466/12 e foi realizada após aprovação do Comitê de Ética em Pesquisa com seres humanos do Centro Universitário de Maringá, mediante parecer $n^{0} 1.445 .307$. Para manter $\mathrm{o}$ anonimato, as mulheres que compuseram a amostra deste estudo foram identificadas pela letra $\mathrm{E}$, que correspondente a letra inicial da palavra entrevistada, seguida de ordenação numérica (E1, E2, ... E16).

\section{RESULTADOS}

Em relação ao perfil das gestantes a Tabela 1 apresenta os dados relativos à díade, à escolaridade e ao estado civil, assim como a idade gestacional (IG) em que foi realizado o parto das mulheres entrevistadas.

Foi possível identificar no discurso das entrevistas duas categorias de análise: a expectativa do parto no terceiro trimestre de gestação e a experiência do parto.

Em relação à expectativa do parto no terceiro trimestre os achados do presente estudo evidenciaram que o parto normal é a via de parto mais desejada pelas gestantes, pois das 16 entrevistadas nove relataram preferência pela via de parto normal e seis pelo parto cirúrgico, conforme apresentado na Tabela 2.

A experiência do parto relatada pelas entrevistadas permitiu identificar que dez mulheres vivenciaram o parto desejado, entretanto foi realizado parto cirúrgico em cinco das nove mulheres que elegeram o parto normal, conforme apresentado na Tabela 3. 
Tabela 1. Características das participantes em relação aos dados sociodemográficos e à idade gestacional do parto, Maringá, PR, Brasil, 2017

\begin{tabular}{ccccc}
\hline Participantes & Idade & Escolaridade & Estado civil & IG do parto \\
\hline E1 & 28 & Ensino médio & Casada & $39 \mathrm{~s} 5 \mathrm{~d}$ \\
E2 & 18 & Ensino fundamental & Solteira & $38 \mathrm{~s}$ \\
E3 & 24 & Ensino fundamental & Casada & $40 \mathrm{~s}$ \\
E4 & 21 & Ensino médio & Casada & $40 \mathrm{~s}$ \\
E5 & 34 & Ensino médio & Casada & $38 \mathrm{~s} 1 \mathrm{~d}$ \\
E6 & 33 & Ensino fundamental & União estável & $38 \mathrm{~s}$ \\
E7 & 31 & Ensino médio & Casada & $37 \mathrm{~s}$ \\
E8 & 29 & Ensino médio & União estável & $41 \mathrm{~s} 2 \mathrm{~d}$ \\
E9 & 28 & Ensino fundamental & União estável & $38 \mathrm{~s} 1 \mathrm{~d}$ \\
E10 & 20 & Ensino médio & Casada & $38 \mathrm{~s}$ \\
E11 & 18 & Ensino médio & Casada & $40 \mathrm{~s}$ \\
E12 & 21 & Ensino médio & Casada & $40 \mathrm{~s}$ \\
E13 & 24 & Ensino médio & Casada & $38 \mathrm{~s} 6 \mathrm{~d}$ \\
E14 & 22 & Ensino médio & Casada & $39 \mathrm{~s} 5 \mathrm{~d}$ \\
E15 & 29 & Ensino médio & Casada & $38 \mathrm{~s} 2 \mathrm{~d}$ \\
E16 & 23 & Ensino médio & Casada & 38 \\
\hline
\end{tabular}

Fonte: Elaborado pela autora, com base na pesquisa. *s: semanas *d: dias

Tabela 2. Via de parto desejada pelas mulheres entrevistadas no $3^{\circ}$ trimestre de gestação, Maringá, PR, Brasil, 2017

\begin{tabular}{l|c}
\hline Variáveis & Gestantes \\
\hline Parto normal & 9 \\
Parto cesáreo & 6 \\
Não sabia & 1 \\
\hline Total & 16 \\
\hline
\end{tabular}

Fonte: Elaborado pela autora, com base na pesquisa.

Tabela 3. Via de parto desejada e desfecho do parto, Maringá, PR, Brasil, 2017

\begin{tabular}{cccc}
\hline \multirow{2}{*}{ Parto desejado } & N 16 & Parto realizado \\
\cline { 3 - 4 } Parto normal & 9 & 5 & Cesárea \\
Parto cesáreo & 6 & 1 & 4 \\
Não sabia & 1 & - & 5 \\
\hline
\end{tabular}

Fonte: Elaborado pela autora, com base na pesquisa. 


\section{DISCUSSÃO}

A amostra foi composta por mulheres com idade média de 25 anos, casadas e com relacionamento estável. Quanto à escolaridade, predominou o ensino médio completo (12) seguido do ensino fundamental (4). Considerando que o baixo grau de escolaridade é indicado como um dos itens de avaliação da estratificação de risco durante o pré-natal, tal dado sinaliza a necessidade da observação mais pontual da equipe de saúde diante das demandas destas mulheres ao longo do período gestacional, de acordo com as recomendações do Ministério da Saúde 8 .

Em relação à idade gestacional no momento do parto, observou-se que somente sete das participantes tiveram o desfecho com idade gestacional maior ou igual a 39 semanas, conforme preconizado pela nova resolução $\mathrm{n}^{\circ} 2.144$, de 17 de março de 2016 que estabeleceu a realização da cesariana sem indicações clínicas a partir da $39^{\mathrm{a}}$ semana de gestação ${ }^{14}$. A antecipação das cesáreas eletivas para idades gestacionais entre 37 e 38 semanas tem sido justificada com o argumento de que parto prematuro é aquele realizado antes de 37 semanas $^{15}$.

A realização da cesariana eletiva com 37 ou 38 semanas de idade gestacional, apesar de frequente na prática médica, não encontra apoio na literatura científica que, por sua vez determina que a cesárea eletiva seja realizada após as 39 semanas de gestação $0^{15}$.

\section{A EXPECTATIVA DO PARTO NO TERCEIRO TRIMESTRE DE GESTAÇÃO}

A preferência das mulheres pela via de parto normal relatada neste estudo foi descrita anteriormente em um estudo nacional realizado com mais de 23 mil puérperas, que revelou a preferência de $66 \%$ das participantes pelo parto normal, o que corrobora com os achados deste estudo ${ }^{16}$. Também foi relatado que as gestantes primíparas têm a preferência maior pelo parto normal, e o principal motivo sinalizado é a recuperação mais rápida ${ }^{16}$, como neste estudo.

As gestantes que pretendiam o parto normal foram arguidas sobre o motivo que as levaram a escolher esta via de parto e as narrativas revelaram a rápida recuperação que proporciona o bem-estar materno para amamentar e para realizar os cuidados do bebê, conforme nos seguintes fragmentos:

Porque sente dor só na bora né e na cesárea não, na cesárea se fica lá... fica sofrendo. (E4)

Pela recuperação né, eu acho que o parto normal é mais seguro né para criança tanto para mãe (E3)

Além da recuperação, é a amamentação, é mais fácil para você cuidar do bebê. (E8)

Estudos anteriores descreveram que o parto normal foi a via de parto escolhida pelas gestantes, em detrimento do parto cirúrgico, por acreditarem que a via vaginal garante a saúde materna e do recém-nascido ${ }^{16}$. As gestantes consideraram vários fatores que repercutem na escolha do parto normal, dentre eles destaca-se a rápida reabilitação da mulher para que possa realizar suas atividades domésticas no período do puerpério, a contribuição para a experiência de ser mãe favorecendo a interação com o bebê, $o$ fato de não ser submetida a uma intervenção cirúrgica, os menores níveis de dor no pós-parto e o entendimento de que a via de parto normal é natural ${ }^{16,17}$.

A expectativa de estar bem após o parto para suprir as necessidades do seu filho, que para elas irá depender totalmente dos seus cuidados, é percebido como uma das principais justificativas para a escolha do parto normal. O motivo principal das mulheres na escolha da via de parto é encontrar-se com saúde no pós-parto para poder cuidar do recém-nascido, visto que o parto normal proporciona melhor qualidade da relação do binômio mãe-bebê por possibilitar maior protagonismo da mulher ${ }^{17}$.

Em relação às gestantes que apontaram o desejo de parto cesariana, notou-se que as expectativas foram permeadas principalmente pelo medo da dor e do sofrimento, como pode-se observar:

Eu tenho preferência pela cesárea porque eu tenbo um probleminba de dor eu sou intolerante a dor e se eu sentir dor intensa eu desmaio então eu, eu não consigo ter um parto normal, mas se eu pudesse optar pelo parto normal eu teria com certeza. (E1) É eu quero pretendo ter cesárea pelo fato 
de eu ter medo do parto normal, muito medo, que eu acho que 99\% das mulberes tem né, mais eu sou bem medrosa, então, por enquanto é cesárea, a tendência tá pesando pra cesárea. (E7)

Ai minhas amigas falam né que elas tiveram normal e... sofreram muito né, elas falam que se fosse boje elas não teriam, elas preferiam fazer cesárea daí por isso eu fiquei bem com medo (E9).

O desfecho gestacional é associado por muitas mulheres ao medo, a provação e ao sofrimento, nas mais diversas culturas e grupos sociais, assim, o medo de não suportar a dor durante o trabalho de parto e a busca de ter uma experiência positiva do parto influenciam na incidência de partos cirúrgicos. A escolha pela cesariana é descrita em alguns estudos como uma opção de favorecimento ao alívio da dor e do medo ${ }^{16-18}$. Associado ao medo da dor existe a disseminação da ideia de que o parto normal apresenta risco para o bebê como lesões anatômicas, o que contribui para que as gestantes optem pelo parto cirúrgico, mesmo sem indicação obstétrica para esse procedimento, denominado de cesárea a pedido ou eletiva ${ }^{19}$.

O planejamento da gestação é indicado como um fator que leva a mulher a escolher o parto cesáreo, visto que buscam informações acerca da via de parto e identificam a cesariana como mais segura para o nascimento do seu filho ${ }^{20}$. Além do medo da dor do parto normal o pedido ao médico para a realização da cesariana ocorre também em função da superestimação da segurança que o uso da tecnologia oferece para a saúde materno-infantil e da subestimação dos riscos da cesárea, pelo medo do parto normal comprometer a anatomia da vagina, desconhecimento dos benefícios do parto normal, experiências prévias com o parto, história e influência familiar, com destaque para a figura materna e falta de informação na assistência pré-natal ${ }^{21,22}$.

Os profissionais de saúde devem realizar a assistência pré-natal baseados nos princípios da humanização, acolhendo a mulher na sua singularidade e realizando a escuta dos seus medos e temores pode promove a criação do vínculo entre o profissional e a gestante e diminuir o sofrimento gerado pelas expectativas em relação ao parto ${ }^{23}$. O estudo relatou o alto nível de medo do desfecho gestacional que resulta em sofrimento para a gestante e interfere de forma negativa em sua saúde mental ${ }^{24}$.

As participantes relataram, durante a entrevista, que a construção da expectativa pela via de parto teve participação direta do cônjuge, familiares, vínculo social e da mídia. As mães e as amigas foram as pessoas mais influentes nesta decisão, visto que as experiências de outras mulheres serviram como informação e esclarecimento de dúvidas:

Sempre tem alguém da família que fala né (E1).

Não tive nenbuma orientação. A minha mãe é que fala para ter parto normal que é bem mais fácil [...] (E4).

Um fator preocupante evidenciado na pesquisa foi que entrevistadas não se referiram às orientações que deveriam ter recebido nos atendimentos realizados na assistência pré-natal, portanto as ações de saúde realizadas ao longo do período gestacional não foram suficientes para esclarecer sobre as vias de parto contribuindo para um entendimento adequado, pois todas as entrevistadas deste estudo relataram a necessidade de orientações sobre o período gravídico puerperal. Assim como descrito na literatura os resultados deste estudo também apontaram a influência da família, prioritariamente da mãe e a falta de preparo e de orientação nos atendimentos realizados no pré-natal ${ }^{21,22}$.

\section{A EXPERIÊNCIA DO PARTO}

Após a realização do parto, as mulheres foram encorajadas a falar sobre a experiência vivida. Por um lado, aquelas que desejaram e realizaram o parto cirúrgico relataram a cesárea como um método doloroso e sofrido, como no excerto:

Assim, foi bom, mas nossa depois a gente fica dependendo dos outros, não consegue nem ir no banbeiro sozinha, dói tudo, parece que a barriga vai abrir[...]nossa é uma sensação muito ruim [...]mas ver a neném bem compensa tudo, todo sofrimento. (E9) 
Por outro lado, o depoimento das entrevistadas que escolheram e tiveram a experiência do parto normal foi de satisfação.

A foi bom [...] por causa do neném, ficou mais perto de mim (E2).

A foi, eu queria mesmo o normal, porque depois que eu vi a cesárea nossa é sofrida e eu já andava por todo bospital (E3).

Totalmente diferente, assim eu imaginava uma coisa imaginava que doía que seria uma coisa assim, mas eu acho que foi falta de, de alguém me orientar melhor lá, acho que foi isso porque se tivesse me orientado melhor ou tivesse [...]ó vai demorar, eu não imaginava que fosse tantas horas, tirando a dor, mas que é uma dor normal, faz parte [...] ver aquela coisinba linda vale a pena (E8).

Outro fato interessante é que as puérperas que relataram os aspectos negativos do pós-parto cirúrgico recomendariam essa via de parto a outras mulheres, denunciando a cultura da cesárea em nosso país. Estudos demonstraram que as mulheres multíparas repetem a experiência do parto anterior, principalmente aquelas que realizaram o parto cirúrgico, o que justifica os indicadores que apontam que a cesárea é mais prevalente em mulheres com gravidez anterior ${ }^{16}$.

A realização do parto cirúrgico não deve ser entendida como uma intervenção cirúrgica trivial, pois os riscos relacionados a esse procedimento sem uma real necessidade não justificam a sua realização. A cesariana tem como principais consequências o aumento de risco de hemorragia pós-parto, necessidade de internação em Unidade de Terapia Intensiva, ruptura uterina em gestações subsequentes com placentação anormal e mortalidade materna. Os riscos relacionados ao recémnascido compreendem as complicações potenciais incluindo o aumento da necessidade de suporte ventilatório no nascimento e maior uso de Unidade de Terapia Intensiva neonata ${ }^{12}$.

A assistência deve ser baseada no atendimento humanizado e assegurar o bem-estar da gestante no desfecho gestacional. As mulheres que realizaram parto normal reviveram a experiência do nascimento do seu bebê com satisfação.
Dói, mas tudo vale a pena quando você vê seu bebê bem, e nossa é só na hora, depois é muito bom (E12).

Foi, foi melhor que eu pensava, a dor nem é assim tão insuportável, dói, mas depois é muito bom, eu andava cuidava do neném enquanto as meninas do quarto (que fizeram cesárea) nossa sofria, eu que ajudava elas (E14).

O parto normal é mais saudável tanto para a mãe quanto para o bebê, pois os benefícios psicológicos para a mãe após o parto são significativos e a satisfação pessoal com o nascimento do filho(a), o bem-estar após o parto, a alegria e a felicidade por estar apta para cuidar do seu bebê foi descrita na literatura ${ }^{25}$, o que corrobora com os achados deste estudo.

Com relação à experiência do parto, seis mulheres não vivenciaram o parto pretendido, sendo que das nove gestantes que relataram preferência pelo parto normal somente em cinco a via de parto foi normal, o parto das outras quatro mulheres foi cirúrgico. Os altos índices de parto cirúrgico vêm aumentando significativamente nas últimas décadas, principalmente nas regiões com elevado índice de desenvolvimento humano ${ }^{8}$.

Entre as que almejavam o parto normal $\mathrm{e}$ realizaram cesárea foi notório o sentimento de frustração, conforme os fragmentos abaixo:

Judiaram de mim, se não fosse fazer barraco lá [...] Mas é muito ruim depois nossa (E4).

Nossa fiquei tão triste, mas o médico achou melhor, daí foi cesárea mesmo[...] Não foi bom (E13).

O sofrimento retratado pelas entrevistadas sinaliza a fragilidade e a vulnerabilidade que permeiam o momento do parto, vários fatores podem interferir no modo que a mulher o enfrentará, podendo tornar a experiência extremamente penosa para a mulher. A insatisfação quanto à assistência recebida durante o trabalho de parto, a presença ou não de um acompanhante, sensações de dor e medo são fatores marcantes do desfecho gestacional ${ }^{25,26}$.

O Ministério da Saúde, em 2016, implantou o Protocolo Clínico de Diretrizes Terapêuticas para Cesariana, orientando sobre os parâmetros que 
necessitam ser observados e seguidos pelas Secretarias de Saúde dos Estados, Distrito Federal e municípios, cujas diretrizes conduzem a assistência obstétrica para uma linha de práticas humanizadas a fim de derrubar o mito de que a cesariana é mais segura que o parto normal. O mesmo documento estabeleceu a obrigatoriedade do esclarecimento à gestante sobre os potenciais riscos e eventos adversos relacionados ao procedimento cirúrgico e uso dos medicamentos para a operação cesariana, entretanto nas maternidades é solicitada apenas a assinatura da gestante ou responsável. ${ }^{7}$

Em consonância com o Programa de Humanização do Pré-natal e Nascimento ${ }^{27}$, as diretrizes do Protocolo Clínico de Diretrizes Terapêuticas para Cesariana preconizou a assistência integral e qualificada na gestação, parto e puerpério, para diminuir o percentual de partos cirúrgicos realizados e restringir a utilização de práticas inadequadas que pudessem comprometer a saúde da mulher e do bebê. Assim como o acolhimento e as práticas humanizadas na atenção obstétrica e neonatal como elemento primordial para o adequado acompanhamento do parto e puerpério. ${ }^{28}$ Entretanto, é necessário o direcionamento dos serviços de saúde nesta direção consolidando as novas práticas no atendimento em saúde.

Frente a fala da única mulher que não vivenciou o parto cesáreo desejado, pode-se inferir que o medo que permeava a possibilidade de vivenciar esse parto foi transformado em força e satisfação para a chegada de seu bebê, conforme o seu depoimento:

É eu tinha muito medo porque eu sempre fui intolerante a dor ne a dor dai eu desmaio [...] mas nossa foi tudo bem, eu fui muito bem atendida, muito. A enfermeira que me atendeu é muito paciente coitada, acho que eu dei bastante trabalbo [...] olba eu tive muita dor, mas faz parte né então, foi ótimo, valeu a pena porque depois, é como todo mundo fala, depois é bem melhor a recuperação e realmente foi (E1).

Embora não tenha sido a via de parto preferida pela gestante a experiência da dor do parto normal foi narrada como algo que fez parte do processo sublimando o medo e transformando a mulher em protagonista do nascimento do seu bebê. A dor do parto normal foi compreendida por mulheres em outros estudos como tolerável e a preferência e/ou satisfação com a via de parto vaginal foi relacionada com o autocuidado e com a rápida e indolor recuperação no pós-parto, como neste estudo ${ }^{29}$.

O medo do desfecho gestacional foi descrito como fobias do parto que afloram principalmente no terceiro trimestre de gestação, tanto em relação a via normal quanto a cirúrgica, sendo associado a vários fatores, entre eles a dor durante o trabalho de parto normal e após o parto quando o desfecho for a cesariana, o medo da anestesia, o medo de não saber ou não conseguir dar à luz, ou ainda, de não viver seu parto de forma natural ${ }^{26}$.

A falta de esclarecimento por parte dos profissionais de saúde sobre as vias de parto foi observada neste estudo, pois quando as entrevistadas foram incentivadas a falar sobre os motivos que a levaram a escolher determinado tipo de parto as explicações foram vagas e superficiais. Este resultado aponta a necessidade de uma comunicação mais efetiva entre a equipe de saúde e as gestantes, pois estudo realizado, anteriormente, a expectativa de gestantes e de obstetras na escolha da via de parto foi diferente, visto que os médicos preferiram a cesariana enquanto as gestantes o parto normal ${ }^{18}$.

A resolução $\mathrm{n}^{\circ} 2.144$, de 17 foi publicada em março de 2016 e enfatizou a importância da ética médica no sentido de esclarecer a gestante sobre o desfecho gestacional, portanto nesta resolução ficou estabelecido o direito da gestante de receber informações sobre os riscos e os benefícios tanto do parto vaginal quanto cirúrgico. Foi estabelecido também que para garantir a segurança do feto, a cesariana só pode ser realizada a partir da $39^{a}$ semana de gestação, devendo ser registrada sua justificativa no prontuário do paciente ${ }^{14}$.

Uma das participantes não realizou a segunda etapa da entrevista, entretanto algumas informações foram obtidas por contato telefônico. Foi realizado parto cirúrgico com 38 semanas de gestação, o bebê se encontrava bem, relatou não ter vivenciado o parto que desejava e alguns sintomas de depressão. Por limitações de dados, não foi possível identificar se houve indicação clínica para a realização da cesariana e a Unidade Básica de Saúde não tinha informações sobre o quadro depressivo desta mulher até o momento do contato telefônico. 
A depressão pós-parto foi pesquisada em uma amostra com características semelhantes à população do presente estudo e os resultados demonstraram ambivalência de sentimentos materno e ansiedade diante das demandas do bebê por cuidados, dificuldades relacionadas à amamentação e instabilidades no sono do bebê, o que revela a qualidade da relação mãe-bebê. ${ }^{30}$ A depressão pós-parto causa grande sofrimento na mulher, especialmente quando se depara com a rejeição e agressividade manifestada contra o bebê e, diante da dualidade de sentimentos, a criança fica vulnerável aos cuidados ou maus-tratos maternos.

\section{CONCLUSÃO}

A cesárea eletiva foi a principal via de parto realizada pelos obstetras, mesmo em mulheres cuja preferência era a via de parto vaginal, sendo realizada inclusive com 37 ou 38 semanas de idade gestacional contrariando a literatura médica que, por sua vez recomenda que seja realizada somente após a $39^{a}$ semana de gestação. Esta recomendação baseia-se no aumento das complicações neonatais que podem resultar em internação em unidade de terapia intensiva neonatal, sepse e parada respiratória por antecipação do momento do parto, sem indicação obstétrica.

Concluiu-se que é preciso investir no esclarecimento da população médica, das gestantes e dos seus familiares de que a cesárea eletiva expõe desnecessariamente os recém-nascidos a riscos e complicações neonatais que podem deixar sequelas, assim como na implementação do Protocolo Clínico de Diretrizes Terapêuticas para Cesariana nas maternidades do Sistema Único de Saúde e da Saúde Suplementar.

Apesar das inegáveis mudanças ocorridas no âmbito da saúde da mulher, a atenção e a humanização do cuidado no ciclo gravídico-puerperal ainda se constitui em um grande desafio. Portanto, é necessária a elaboração de programas e de ações de promoção à saúde do grupo materno-infantil capacitando os profissionais que compõem as equipes da estratégia da saúde da família e a população, favorecendo o cuidado e o autocuidado das mulheres ao longo do período gestacional, parto e puerpério.

É importante destacar que o estudo qualitativo visa compreender os fenômenos a partir da perspectiva dos participantes, portanto algumas limitações devem ser consideradas. A amostra foi composta por mulheres jovens atendidas em Unidade Básica de Saúde do Sistema Único de Saúde, as quais possuem características próprias, cujo processo de seleção não teve como foco a representatividade estatística.

\section{REFERÊNCIAS}

1. Domingues RMSM, Hartz ZM de A, Dias MAB, Leal M do C. Avaliação da adequação da assistência prénatal na rede SUS do Município do Rio de Janeiro, Brasil. Cad Saúde Públ. 2012;28(3):425-37.

2. Cunha ACB da, Santos C, Gonçalves RM. Concepções sobre maternidade, parto e amamentação em grupo de gestantes. Arq Bras Psicol [Internet]. 2012;64(1):139-55.

3. Gama A de S, Giffin KM, Angulo-Tuesta A, Barbosa GP, D'Orsi E. Representações e experiências das mulheres sobre a assistência ao parto vaginal e cesárea em maternidades pública e privada. Cad Saúde Públ. 2009;25(11):2480-8.

4. Zanardo GL de P, Uribe MC, Nadal AHR De, Habigzang LF. Violência obstétrica no Brasil: uma revisão narrativa. Psicol Soc. 2017;29:1-11.

5. Pasche DF, de Albuquerque Vilela ME, Martins CP. Humanização da atenção ao parto e nascimento no Brasil: pressupostos para uma nova ética na gestão e no cuidado. Rev Tempus Actas Saúde Coletiva. 2010;4(4):105-17.

6. Mandarino NR, Chein $\mathrm{MB}$ da C, Monteiro Júnior F das C, Brito LMO, Lamy ZC, Nina VJ da S, et al. Aspectos relacionados à escolha do tipo de parto: um estudo comparativo entre uma maternidade pública e outra privada, em São Luís, Maranhão, Brasil. Cad Saúde Públ. 2009;25(7):1587-96.

7. Brasil. Ministério da Saúde. Diretrizes de Atenção à Gestante: a operação cesariana. Ministério da Saúde. Brasilia, Distrito Federal. 2016.

8. Brasil. Ministério da Saúde. Informações de Saúde Estatísticas Vitais [Internet]. DATASUS. Ministério da Saúde. Brasilia, Distrito Federal. 2016.

9. Organização Mundial da Saúde. Declaração da Organização Mundial da Saúde sobre Taxas de Cesáreas. Human Reproduction Programme. Word Health Organization. Genebra, Suíca. 2015. 
10. Brasil. Ministério da Saúde. Diretrizes nacionais de assistência ao normal parto: Versão resumida. Ministério da Saúde. Brasilia, Distrito Federal. 2017.

11. Andrade P de ON, Silva JQP da, Diniz CMM, Caminha M de FC. Fatores associados à violência obstétrica na assistência ao parto vaginal em uma maternidade de alta complexidade em Recife, Pernambuco. Rev Bras Saúde Matern Infant. 2016;16(1):29-37.

12. Weidle WG, Medeiros CRG, Grave MTQ, Dal Bosco SM. Escolha da via de parto pela mulher: autonomia ou indução? Cad Saúde Col. 2014;22(1):46-53.

13. Bardin L. Análise de Conteúdo. São Paulo: Edições $70 ; 2011$.

14. Brasil. Ministério da Saúde. Secretaria de Atenção à Saúde Departamento de Atenção Básica. Caderno de Atenção Básica: Atenção ao Pré-Natal de Baixo Risco. Ministério da Saúde. Brasilia, Distrito Federal. 2012.

15. Francisco RPV, Zugaib M. Intercorrências neonatais da cesárea eletiva antes de 39 semanas de gestação. Rev Assoc Med Bras. 2013;59(2):93-4.

16. Domingues RMSM, Dias MAB, Nakamura-Pereira M, Torres JA, D'Orsi E, Pereira APE, et al. Processo de decisão pelo tipo de parto no Brasil: da preferência inicial das mulheres à via de parto final. Cad Saúde Públ. 2014;30(1):101-16.

17. Benute GRG, Nomura RY, Santos AM dos, Zarvos MA, Lucia MCS de, Francisco RPV. Preferência pela via de parto: uma comparação entre gestantes nulíparas e primíparas. Rev Bras Ginecol e Obs. 2013;35(6):281-5.

18. Junior TL, Steffani JA, Bonamigo EL. Escolha da via de parto: expectativa de gestantes e obstetras. Rev Bioética. 2013;21(3):509-17.

19. Tostes NA, Seidl EMF. Expectativas de gestantes sobre o parto e suas percepções acerca da preparação para o parto. Temas em Psicol. 2016;24(2):681-93.

20. Nunes ACF, Ramos DKR, Mesquita SK da C. Preferência por cesarianas em gestantes nulíparas de um consultório particular de ginecologia e obstetrícia: um estudo de caso. Rev da Univ Val do Rio Verde. 2014;12(2):743-53.

21. Silva SPC e, Prates RDCG, Campelo BQA. Parto normal ou cesariana? Fatores que influenciam na escolha da gestante. Rev Enferm da UFSM. 2014;4(1):1-9.

22. Santana FA, Lahm JV, dos Santos RP. Fatores que influenciam a gestante na escolha do tipo de parto. Rev da Fac Ciências Médicas Sorocaba. 2015;17(3):123-7.

23. Araujo SM, Silva MED, Moraes RC, Alves DS. A importância do pré-natal e a assistência de enfermagem. VEREDAS FAVIP-Rev Elet de Ciên [Internet]. 2010;3(2):7.

24. Veringa IK, de Bruin EI, Bardacke N, Duncan LG, van Steensel FJA, Dirksen CD, et al. Ive Changed My Mind, Mindfulness-Based Childbirth and Parenting (MBCP) for pregnant women with a high level of fear of childbirth and their partners: study protocol of the quasi-experimental controlled trial. BMC Psychiatry. 2016;16(1):377.

25. Oliveira ASS, Rodrigues DP, Guedes MVC, Felipe GF. Percepção de mulheres sobre a vivência do trabalho de parto e parto. Rev da Rede Enferm do Nord. 2010;11:32-41.

26. Szejer M, Benetti MNB, Stewart R, Lambrichs LL, Frydman R. Nove meses na vida da mulher: uma abordagem psicanalítica da gravidez e do nascimento. Casa do Psicólogo; 1997. 322 p.

27. Brasil. Ministério da Saúde. Programa Humanização do Parto - Humanização no Pré-Natal e no Nascimento. Ministério da Saúde. Brasilia, Distrito Federal. 2002.

28. Vieira MJ de O, Santos AAP dos, Silva JM de O e, Sanches MET de L. Assistência de enfermagem obstétrica baseada em boas práticas: do acolhimento ao parto. Rev Eletrônica Enferm. 2016;18:10.

29. Reis CC, de Souza KRF, Alves DS, Tenório IM, Neto WB. Percepção das mulheres sobre a experiência do primeiro parto: implicações para o cuidado de enfermagem. Cienc y Enfermería. 2017;23(2):4556.

30. Greinert BRM, dos Reis Carvalho E, Capel H, Marques AG, Milani RG. A relação mãe-bebê no contexto da depressão pós-parto: estudo qualitativo. Saúde e Pesqui. 2018;11(1):81-8.

Recebido em: 18/07/2019

Aceito em: 11/09/2019 